\title{
Effect of MRI acquisition acceleration via compressed sensing and parallel imaging on brain volumetry
}

\author{
Michael Dieckmeyer ${ }^{1}$ (1) $\cdot$ Abhijit Guha Roy $^{2} \cdot$ Jyotirmay Senapati $^{2} \cdot$ Christian Wachinger $^{2} \cdot$ Lioba Grundl $^{1}$. \\ Jörg Döpfert ${ }^{3} \cdot$ Pere Ferrera Bertran $^{3} \cdot$ Andreas Lemke $^{3}$. Claus Zimmer ${ }^{1}$. Jan S. Kirschke ${ }^{1} \cdot$ Dennis M. Hedderich ${ }^{1}$
}

Received: 15 April 2020 / Revised: 15 December 2020 / Accepted: 30 December 2020 / Published online: 27 January 2021

(c) The Author(s) 2021

\begin{abstract}
Objectives To investigate the effect of compressed SENSE (CS), an acceleration technique combining parallel imaging and compressed sensing, on potential bias and precision of brain volumetry and evaluate it in the context of normative brain volumetry.

Materials and methods In total, 171 scans from scan-rescan experiments on three healthy subjects were analyzed. Each subject received 3D-T1-weighted brain MRI scans at increasing degrees of acceleration (CS-factor = 1/4/8/12/16/20/32). Single-scan acquisition times ranged from 00:41 $\mathrm{min}(\mathrm{CS}$-factor $=32)$ to 21:52 $\mathrm{min}(\mathrm{CS}$-factor $=1)$. Brain segmentation and volumetry was performed using two different software tools: $m d$.brain, a proprietary software based on voxel-based morphometry, and FreeSurfer, an open-source software based on surface-based morphometry. Four sub-volumes were analyzed: brain parenchyma (BP), total gray matter, total white matter, and cerebrospinal fluid (CSF). Coefficient of variation (CoV) of the repeated measurements as a measure of intra-subject reliability was calculated. Intraclass correlation coefficient (ICC) with regard to increasing CS-factor was calculated as another measure of reliability. Noise-to-contrast ratio as a measure of image quality was calculated for each dataset to analyze the association between acceleration factor, noise and volumetric brain measurements.

Results For all sub-volumes, there is a systematic bias proportional to the CS-factor which is dependent on the utilized software and subvolume. Measured volumes deviated significantly from the reference standard (CS-factor $=1$ ), e.g. ranging from 1 to $13 \%$ for BP. The CS-induced systematic bias is driven by increased image noise. Except for CSF, reliability of brain volumetry remains high, demonstrated by low $\operatorname{CoV}(<1 \%$ for CS-factor up to 20$)$ and good to excellent ICC for CS-factor up to 12.
\end{abstract}

Conclusion CS-acceleration has a systematic biasing effect on volumetric brain measurements.

Keywords Brain $\cdot$ Magnetic resonance imaging $\cdot$ Acceleration $\cdot$ Systematic bias

Supplementary Information The online version contains supplementary material available at https://doi.org/10.1007/s1033 4-020-00906-9.

Michael Dieckmeyer

michael.dieckmeyer@tum.de

1 Department of Diagnostic and Interventional Neuroradiology, Klinikum Rechts Der Isar der Technischen Universität München, Ismaninger Str. 22, 81675 Munich, Germany

2 Lab for Artificial Intelligence in Medical Imaging, Klinik und Poliklinik Für Kinder- und Jugendpsychiatrie, Psychosomatik und Psychotherapie, Klinikum der Universität München, LMU München, Waltherstr. 23, 80337 Munich, Germany

3 mediaire GmbH, Möckernstraße 63, 10965 Berlin, Germany

\section{Abbreviations}

AP Anterior-posterior direction

BP Brain parenchyma (combined gray and white matter)

$\mathrm{CoV}$ Coefficient of variation

CS Compressed SENSE

CS-factor Compressed SENSE reduction factor

CSF Cerebrospinal fluid

FH Feet-head direction

FS FreeSurfer segmentation and volumetry tool

GM Gray matter

GRAPPA Generalized autocalibrating partially parallel acquisition

ICC Intraclass correlation coefficient

$\mathrm{ICC}_{1, i} \quad$ ICC between CS-factors 1 and $i$ 


$\begin{array}{ll}\text { IRB } & \text { Institutional review board } \\ \text { MDB } & \text { Md.brain segmentation and volumetry tool } \\ \text { ml } & \text { Milliliter } \\ \text { MRI } & \text { Magnetic resonance imaging } \\ \text { NBV } & \text { Normative brain volumetry } \\ \text { NCR } & \text { Noise-to-contrast-ratio } \\ \text { PI } & \text { Parallel imaging } \\ \text { R } & \text { Pearson correlation coefficient } \\ \text { RL } & \text { Right-left direction } \\ \text { SD } & \text { Standard deviation } \\ \text { SENSE } & \text { Sensitivity encoding } \\ \text { SPM } & \text { Statistical parametric mapping } \\ \text { T } & \text { Tesla } \\ \text { T1w } & \text { T1-weighted } \\ \text { TE } & \text { Time of echo } \\ \text { TFE } & \text { Turbo field echo } \\ \text { TR } & \text { Time of repetition } \\ \text { VBM } & \text { Voxel-based morphometry } \\ \text { WM } & \text { White matter }\end{array}$

\section{Introduction}

Thanks to technical advances in the recent years, wholebrain segmentation and volumetry can be performed fully automated and MRI-based brain volumetry is increasingly used in the clinical setting [1].

Its application has great potential to support disease diagnosis, improve the understanding of pathomechanisms, track disease progression and monitor treatment effects [2]. In addition to mere visual image evaluation, it has the potential to deliver a more precise and objective evaluation of patients with neurodegenerative diseases in clinical practice. One important application is normative brain volumetry (NBV), which compares measured volumes of different brain structures to an age- and gender-adjusted healthy cohort to reveal deviations from normal volumes. Recently, NBV has been shown to improve diagnostic accuracy of neurodegenerative atrophy patterns as well as interrater reliability for detection and differential diagnosis of neurodegenerative diseases [3-6]. It is of note that brain volumetry has a far broader range of applications, such as in the investigation of brain development [7].

Whole brain volumetry is usually based on high-quality three-dimensional T1-weighted magnetization-prepared rapid gradient-echo sequences (MPRAGE) [8]. The necessity to encode a large number of $\mathrm{k}$-space lines as well as the preceding inversion time (TI), necessary for the T1-weighted contrast, results in long scan times at low acceleration factors. This might be problematic for widespread clinical adoption considering the increasing workloads and notoriously tight schedules in radiological departments and practices [9]. The long scan times also increase the susceptibility for motion artifacts leading to reduced image quality and accuracy of brain volumetry.

Recent advances on the acquisition side, such as compressed SENSE (CS), GRAPPA or Wave-CAIPI, aim at significantly reducing scan times [10-13]. CS represents a combination of the parallel imaging (PI) technique SENSE $[14]$ and compressed sensing $[15,16]$. Through the application of sparsity constraints and k-space undersampling, a substantial image acquisition acceleration can be achieved. It was recently shown that the implementation of CS into clinical practice of neuroimaging leads to decreased scan times at preserved visual image quality $[17,18]$. Volumetric brain MRI using ultrafast magnetization-prepared rapid gradient-echo (MP-RAGE) sequences has been evaluated recently. The authors used Wave-Controlled Aliasing in Parallel Imaging (Wave-CAIPI; Siemens) for acquisition acceleration and reported low intra-individual variability as well as comparable morphometric estimates between accelerated and non-accelerated scans [19, 20].

However, it is unknown whether accelerated acquisition through CS impacts whole-brain volumetric measurements. Therefore, the aim of our study was to evaluate the effect of CS on brain volumetry and objective image quality using well-controlled test-retest data from three healthy individuals. Two brain volumetry tools were applied to cover distinct morphometry approaches to evaluate the influence of CS on measured brain volumes in surface-based morphometry and voxel-based morphometry.

\section{Materials and methods}

\section{Study subjects}

Three healthy subjects ( 1 female, 2 males; age $27-31$ years) were recruited for this study and gave informed written consent. The study was approved by the local institutional review board (IRB). Each subject underwent three consecutive MRI scans of the brain on different days within a sevenday period. To minimize changes in brain volume depending on the time of day, all scans were performed between 06:00 a.m. and 08:00 a.m. Each MRI scan followed the same protocol as specified below.

\section{MRI protocol}

Imaging was performed on a 3 T MRI scanner (Philips Ingenia, Philips, Best, The Netherlands) using a 32-channel head coil.

CS with increasing degrees of $\mathrm{k}$-space undersampling was used for image acquisition acceleration. The degree of acceleration is expressed by the compressed SENSE reduction factor (CS-factor). Each imaging dataset was inspected 
Table 1 Acquisition times (in minutes and seconds) of the applied 3D-T1w-TFE sequence for each CS-factor

\begin{tabular}{llllllll}
\hline CS-factor & 1 & 4 & 8 & 12 & 16 & 20 & 32 \\
\hline Acquisition time & $21: 52$ & $05: 18$ & $02: 41$ & $01: 49$ & $01: 22$ & $01: 05$ & $00: 41$ \\
\hline
\end{tabular}

CS compressed SENSE

visually by a radiologist to assure the absence of severe motion artifacts.

The MRI protocol consisted of a three-dimensional T1-weighted turbo field echo sequence (3D-T1w-TFE) which was performed at increasing CS-factors of 1 (no acceleration), 4, 8, 12, 16, 20 and 32. For CS-factor $=1$, the sequence was performed once per scan date, resulting in a total of three identical measurements per subject. For each CS-factor $\geq 4$, the sequence was performed three times per scan date, resulting in a total of nine identical measurements per subject. Thus, in total, 171 3D-T1w datasets were acquired.

The acquisition times for each CS-factor are displayed in Table 1. The total exam time per scan date was $62 \mathrm{~min}$. The other sequence parameters were as follows: acquired voxel size, $1 \times 1 \times 1 \mathrm{~mm}^{3}$; field of view, $250 \mathrm{~mm} \times 250 \mathrm{~mm} \times 180 \mathrm{~mm}$ (feet-head $\times$ anterior-posterior $\times$ right-left; $\mathrm{FH} \times \mathrm{AP} \times \mathrm{RL})$; TR, $600 \mathrm{~ms}$; TE $=28 \mathrm{~ms}$; acquisition plane, sagittal.

\section{Image and data analysis}

Based on the acquired 3D-T1w-TFE datasets, cortical reconstruction and volumetric segmentation was performed with two different processing tools:

(i) The commercially available and CE-certified software-tool md.brain v1.1.1 (mediaire GmbH, Berlin, Germany). The software performs a segmentation of different brain regions based on voxel-based morphometry (VBM) and statistical parametric mapping (SPM) and then determines their volumes [21-24]. Throughout the remainder of this article, this processing tool will be referred to as MDB.

(ii) FreeSurfer image analysis suite v6.0, which is documented and freely available for download online [25, 26]. The software performs a segmentation of different brain regions based on surface-based morphometry according to Fischl et al. [27, 28]. Seven of the 27 datasets that were acquired at CS-factor $=32$ could not be analyzed with FS due to insufficient image quality. The most likely reason is the failed convergence of the internal optimization algorithm of the FS software that uses a set of high-quality reference MRI volumes. Throughout the remainder of this article, this processing tool will be referred to as FS.
The following sub-volumes were analyzed: brain parenchyma (equal to combined total gray and white matter volume; BP), total gray matter (GM), total white matter (WM), and cerebrospinal fluid (CSF).

Mean and standard deviation (SD) of the repeated measurements were calculated for each CS-factor and subvolume. The coefficient of variation $(\mathrm{CoV})$ as a measure of intrasubject reliability was calculated as $\mathrm{CoV}=\mathrm{SD} /$ mean.

Intra-class correlation (ICC) was calculated as another measure of reliability with respect to CS-factor. Because of the presence of systematic bias, we chose ICC $(\mathrm{C}, 1)$ according to the McGraw and Wong notation [29] as the appropriate type of ICC [30]. For each software package and each of the four analyzed brain volumes (BP, WM, GM and CSF), ICC was calculated pairwise to assess reliability between measurements at $\mathrm{CS}=1$ and $\mathrm{CS}=4,8,12,16,20$ and 32, respectively, using the following notation: $\mathrm{ICC}_{1,4}, \mathrm{ICC}_{1,8}$, etc.

The linear relationship between the applied CS-factor and the measured brain volume was assessed by calculating Pearson correlation coefficient $\mathrm{R}$ for both software tools and each subvolume.

Noise-to-contrast ratio (NCR) as a measure of image quality was calculated for each acquired dataset using the CAT12 toolbox by measuring the local standard deviation in the optimized WM segment scaled by the minimum tissue contrast [31].

To assess within-session and across-session reproducibility and the potential averaging effects of within-session data, for each CS-factor $>1$, we calculated mean and SD within a single session (3 repeated scans per session) and across sessions (mean of each session for 3 sessions), respectively.

\section{Statistical analysis}

Mean volumes calculated for different CS-factors were compared using paired two-sided $t$ tests with Bonferroni correction to address multiple comparisons. Cohen's $d$ as measure of effect size was calculated for significant values of relevant comparisons.

Outliers of the volume measures were detected using the Grubbs test and removed [32]. All statistical tests were performed at a significance level $\alpha=0.05$. Statistical analyses were performed using MATLAB (The MathWorks, Natick, USA). 
Fig. 1 Sample axial reconstructions of the acquired 3D-T1-weighted brain images at increasing CS-factors of one of the subjects (enlarged view of the image center below each full FOV image). $C S$ compressed SENSE

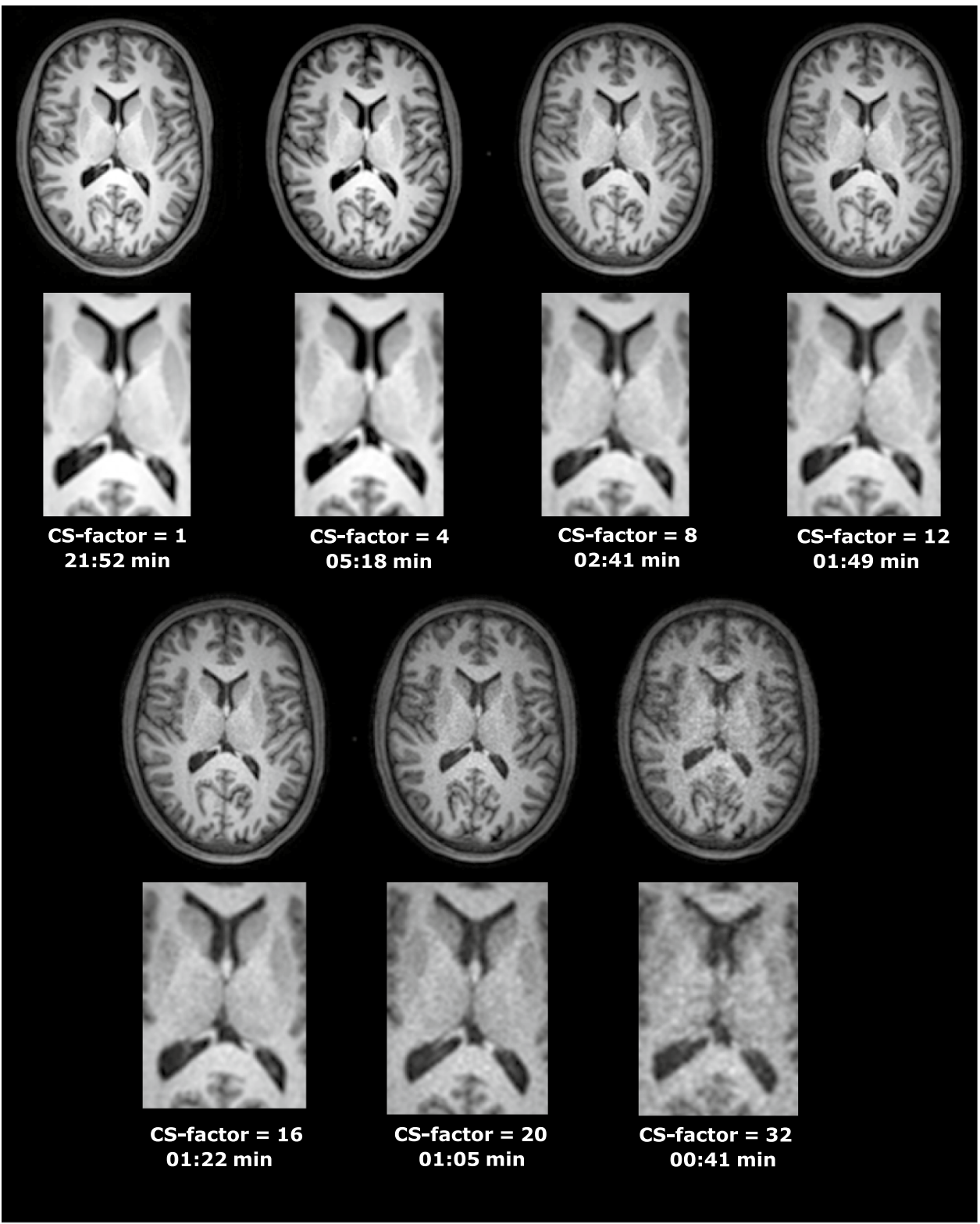

\section{Results}

\section{Measured brain volumes}

Representative axial brain images of WM, GM and CSF at increasing CS-factors of one of the subjects are shown in Fig. 1. Supplementary Figures $2 a(M D B)$ and $2 b$ (FS) display representative axial and coronal segmentation overlays of WM, GM and CSF at increasing CS-factors of one of the subjects. From this figure, it can be appreciated visually how the increasing CS-factor affects the segmentations at the interfaces of the three compartments, in particular for CS-factors $\geq 20$.

Mean and standard deviation of the absolute volumes were calculated for MDB (Fig. 2a) and FS (Fig. 2b).
The analyzed volumes are displayed as a function of the employed CS-factor for each of the three subjects. Calculated volumes relative to the volume at CS-factor $=1$ are shown as mean across all subjects in Fig. 3 a.

For all analyzed sub-volumes and each subject, there is a systematic bias proportional to the employed CS-factor. Degree and direction of the bias are dependent on the utilized processing tool as well as the analyzed sub-volume.

\section{MDB-based volumetry}

For WM, the calculated volumes increase with increasing CS-factor. For all other sub-volumes, the calculated volumes decrease with increasing CS-factor (Fig. 3a). Combining the measured relative volumes of all subjects, the observed 

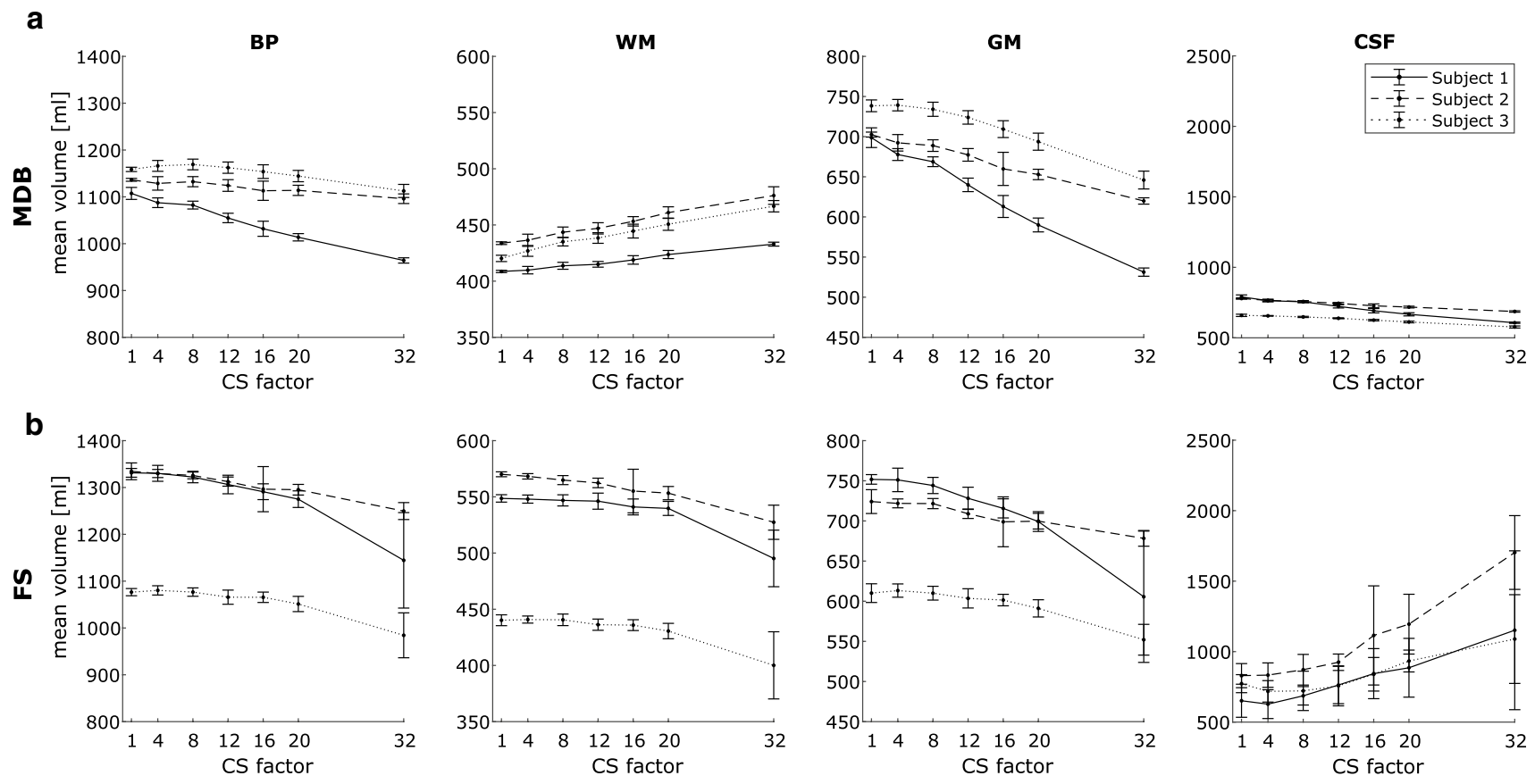

Fig. 2 MDB-based (a, upper row) and FS-based (b, lower row) mean $\pm 2 \mathrm{SD}$ of absolute segmented volumes of the three examined subjects as a function of CS-factor. $C S$ compressed SENSE, $M D B$

md.brain segmentation and volumetry tool, FS FreeSurfer segmentation and volumetry tool, $B P$ brain parenchyma, $W M$ white matter, $G M$ gray matter, $C S F$ cerebrospinal fluid, $S D$ standard deviation
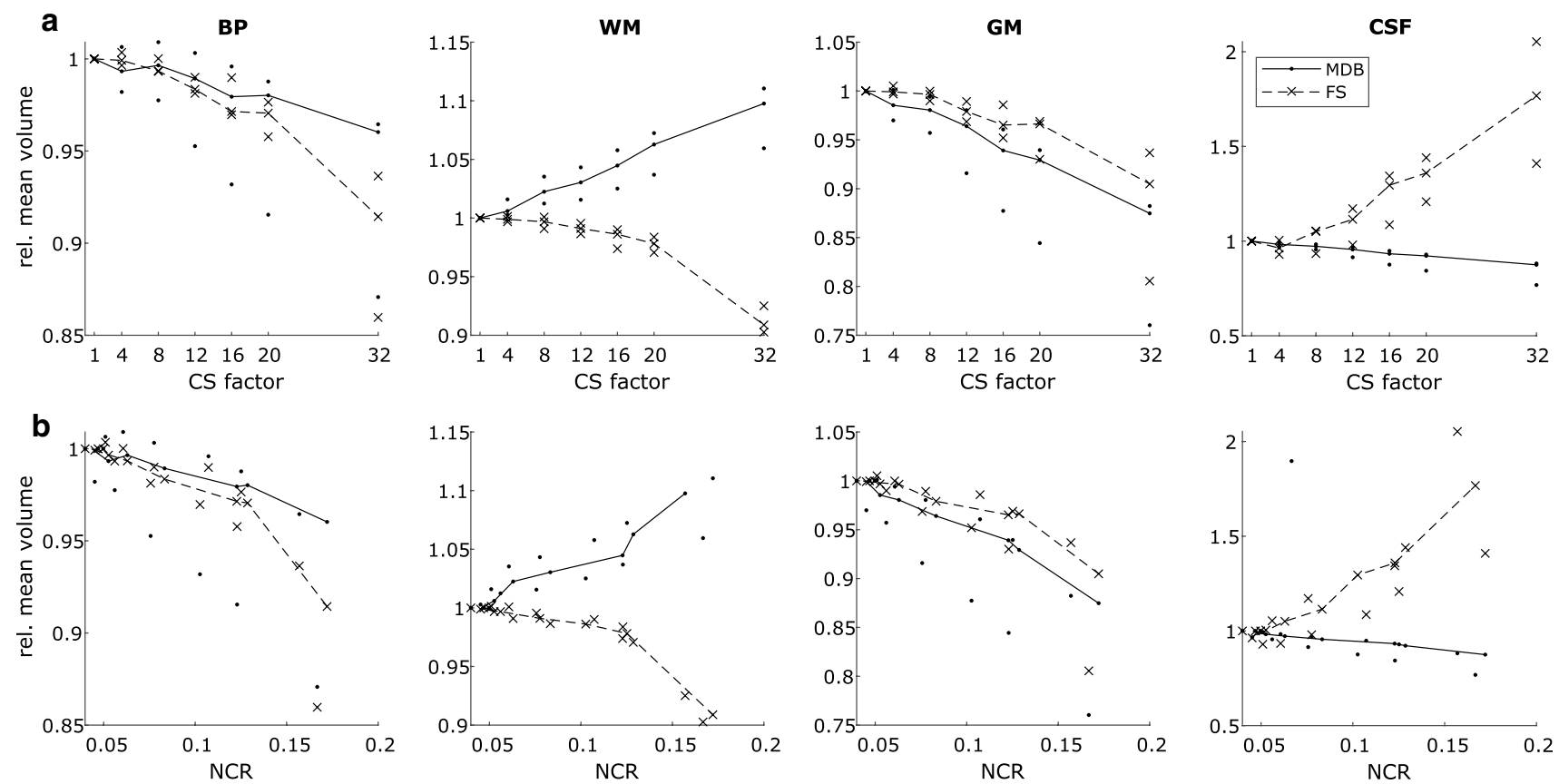

Fig. 3 Segmented volumes relative to the volume at CS-factor $=1$, as a function of CS-factor (a, upper row) and NCR (b, lower row). Each subject is represented by a dot (MDB) or cross (FS) and the median across the three subjects is represented by a solid (MDB) or dashed

(FS) line. $N C R$ noise-to-contrast ratio, $M D B$ md.brain segmentation and volumetry tool, FS FreeSurfer segmentation and volumetry tool, $B P$ brain parenchyma, $W M$ white matter, $G M$ gray matter, $C S F$ cerebrospinal fluid, $C S$ compressed SENSE 
Table 2 MDB-based volumes relative to CS-factor $=1$, mean and SD across all subjects

\begin{tabular}{|c|c|c|c|c|c|c|c|c|c|c|c|c|}
\hline \multirow[t]{2}{*}{ CS-factor } & \multicolumn{2}{|l|}{4} & \multicolumn{2}{|l|}{8} & \multicolumn{2}{|l|}{12} & \multicolumn{2}{|l|}{16} & \multicolumn{2}{|l|}{20} & \multicolumn{2}{|l|}{32} \\
\hline & Mean & SD & Mean & SD & Mean & SD & Mean & SD & Mean & SD & Mean & SD \\
\hline BP & 0.9939 & 0.0114 & 0.9944 & 0.0139 & 0.9817 & 0.0223 & 0.9691 & 0.0286 & 0.9611 & 0.0334 & 0.9318 & 0.0443 \\
\hline GM & 1.0082 & 0.0077 & 1.0234 & 0.0105 & 1.0297 & 0.0125 & 1.0426 & 0.0148 & 1.0573 & 0.0162 & 1.0892 & 0.0229 \\
\hline WM & 0.9855 & 0.0142 & 0.9773 & 0.0164 & 0.9534 & 0.0285 & 0.9257 & 0.0375 & 0.9044 & 0.0438 & 0.8391 & 0.0571 \\
\hline CSF & 0.9811 & 0.0125 & 0.9703 & 0.0121 & 0.9463 & 0.0239 & 0.9190 & 0.0328 & 0.8981 & 0.0398 & 0.8414 & 0.0532 \\
\hline
\end{tabular}

Relative volumes were calculated individually for each subject

$C S$ compressed SENSE, $S D$ standard deviation, $M D B$ md.brain segmentation and volumetry tool, $B P$ brain parenchyma, $W M$ white matter, $G M$ gray matter, $C S F$ cerebrospinal fluid

Table 3 Comparison between MDB-based relative volumes between consecutive CS-factors ( 1 vs. 4,4 vs. 8 , etc.) across all subjects: $p$-values of paired $t$ tests, Cohen's $d$ as measure of effect size

\begin{tabular}{|c|c|c|c|c|c|c|c|c|c|c|c|c|}
\hline \multirow[t]{2}{*}{ CS-factors } & \multicolumn{2}{|l|}{1 vs. 4} & \multicolumn{2}{|l|}{4 vs. 8} & \multicolumn{2}{|l|}{8 vs. 12} & \multicolumn{2}{|l|}{12 vs. 16} & \multicolumn{2}{|l|}{16 vs. 20} & \multicolumn{2}{|l|}{20 vs. 32} \\
\hline & $p$ & $d$ & $p$ & $d$ & $p$ & $d$ & $p$ & $d$ & $p$ & $d$ & $p$ & $d$ \\
\hline BP & 0.010 & 0.53 & 0.591 & - & $<0.001 *$ & 1.28 & $<0.001 *$ & 1.38 & 0.001 & 0.75 & $<0.001 *$ & 2.33 \\
\hline GM & $<0.001 *$ & -1.07 & $<0.001^{*}$ & -2.99 & $<0.001 *$ & -1.14 & $<0.001 *$ & -2.54 & $<0.001 *$ & -2.18 & $<0.001^{*}$ & -3.88 \\
\hline WM & $<0.001 *$ & 1.03 & $<0.001 *$ & 1.75 & $<0.001 *$ & 1.76 & $<0.001 *$ & 2.12 & $<0.001 *$ & 1.45 & $<0.001 *$ & 4.03 \\
\hline $\mathrm{CSF}$ & $<0.001 *$ & 1.51 & $<0.001^{*}$ & 3.14 & $<0.001^{*}$ & 1.82 & $<0.001 *$ & 2.56 & $<0.001 *$ & 1.77 & $<0.001^{*}$ & 3.61 \\
\hline
\end{tabular}

$C S$ compressed SENSE, $M D B$ md.brain segmentation and volumetry tool, $B P$ brain parenchyma, $W M$ white matter, $G M$ gray matter, $C S F$ cerebrospinal fluid

*Marks significant difference to volume at consecutive CS-factor $(p<0.05)$

Table 4 FS-based volumes relative to CS-factor $=1$, mean and SD across all subjects

\begin{tabular}{|c|c|c|c|c|c|c|c|c|c|c|c|c|}
\hline \multirow[t]{2}{*}{ CS-factor } & \multicolumn{2}{|l|}{4} & \multicolumn{2}{|l|}{8} & \multicolumn{2}{|l|}{12} & \multicolumn{2}{|l|}{16} & \multicolumn{2}{|l|}{20} & \multicolumn{2}{|l|}{32} \\
\hline & Mean & SD & Mean & SD & Mean & SD & Mean & SD & Mean & SD & Mean & SD \\
\hline BP & 0.9997 & 0.0056 & 0.9956 & 0.0050 & 0.9849 & 0.0071 & 0.9770 & 0.0144 & 0.9683 & 0.0100 & 0.9133 & 0.0356 \\
\hline GM & 0.9990 & 0.0035 & 0.9962 & 0.0062 & 0.9910 & 0.0064 & 0.9834 & 0.0127 & 0.9775 & 0.0082 & 0.9149 & 0.0248 \\
\hline WM & 1.0004 & 0.0078 & 0.9954 & 0.0073 & 0.9790 & 0.0116 & 0.9677 & 0.0193 & 0.9551 & 0.0196 & 0.8993 & 0.0556 \\
\hline CSF & 0.9657 & 0.0668 & 1.0128 & 0.0885 & 1.0886 & 0.1134 & 1.2417 & 0.1853 & 1.3358 & 0.1527 & 1.7707 & 0.3727 \\
\hline
\end{tabular}

Relative volumes were calculated individually for each subject

$C S$ compressed SENSE, $S D$ standard deviation, $F S$ FreeSurfer segmentation and volumetry tool, $B P$ brain parenchyma, $W M$ white matter, $G M$ gray matter, $C S F$ cerebrospinal fluid

differences between consecutive CS-factors (CS-factor $=1$ vs. 4 , 4 vs. 8 , etc.) were significant $(p<0.05)$ for all subvolumes except for the difference between CS-factors 4 and 8 in BP (Tables 2, 3). The effect size assessed by Cohen's $d$ was larger for MDB than for FS (Tables 3, 5).

\section{FS-based volumetry}

For CSF, the calculated volumes increase with increasing CS-factor. For all other subvolumes, the calculated volumes decrease with increasing CS-factor (Fig. 3a). Combining the measured relative volumes of all subjects, the observed differences between consecutive CS-factors were all significant $(p<0.05)$ for CS-factors $\geq 4$ for all subvolumes (Tables 4, 5).

\section{Noise level analysis}

To obtain a quantitative measure of image quality, we calculated NCR, which increases with more noise added to the images. Pearson correlation coefficient of the CS-factor and the NCR revealed a strong linear correlation $\left(R^{2}=0.98\right.$; see also Suppl. Fig. 1). NCR and the mean of the calculated relative volumes across subjects also showed strong linear correlations which were higher for MDB-based volumes (NCR 
Table 5 Comparison between FS-based relative volumes between consecutive CS-factors ( $\mathrm{CS}=1$ vs. $\mathrm{CS}=4, \mathrm{CS}=4$ vs. $\mathrm{CS}=8$, etc.) across all subjects: $p$-values of paired $t$ tests, Cohen's $d$ as a measure of effect size

\begin{tabular}{|c|c|c|c|c|c|c|c|c|c|c|c|c|}
\hline \multirow[t]{2}{*}{ CS-factors } & \multicolumn{2}{|l|}{1 vs. 4} & \multicolumn{2}{|l|}{4 vs. 8} & \multicolumn{2}{|l|}{8 vs. 12} & \multicolumn{2}{|l|}{12 vs. 16} & \multicolumn{2}{|l|}{16 vs. 20} & \multicolumn{2}{|l|}{20 vs. 32} \\
\hline & $p$ & $d$ & $p$ & $d$ & $p$ & $d$ & $p$ & $d$ & $p$ & $d$ & $p$ & $d$ \\
\hline BP & 0.805 & - & $0.0031 *$ & 0.63 & $<0.001^{*}$ & 2.46 & $0.0047 *$ & 0.60 & $0.0016 *$ & 0.68 & $<0.001 *$ & 1.85 \\
\hline GM & 0.159 & - & $0.0148^{*}$ & 0.50 & $<0.001 *$ & 0.74 & $0.0043^{*}$ & 0.60 & $0.0129 *$ & 0.51 & $<0.001^{*}$ & 2.41 \\
\hline WM & 0.780 & - & $0.0279 *$ & 0.45 & $<0.001^{*}$ & 2.14 & $<0.001 *$ & 0.73 & $0.0013^{*}$ & 0.69 & $<0.001^{*}$ & 1.41 \\
\hline CSF & $0.013^{*}$ & 0.51 & $<0.001 *$ & -0.54 & $0.001 *$ & -0.71 & $<0.001 *$ & -0.90 & $0.0151 *$ & -0.50 & $<0.001 *$ & -1.19 \\
\hline
\end{tabular}

$C S$ compressed SENSE, FS FreeSurfer segmentation and volumetry tool, $B P$ brain parenchyma, $W M$ white matter, $G M$ gray matter, $C S F$ cerebrospinal fluid

*Marks significant difference to volume at subsequent CS-factor $(p<0.05)$

Table 6 Coefficients of variation $(\mathrm{CoV})$ in \% of the MDB-based repeated volumetric measurements

\begin{tabular}{lllllllll}
\hline CS-factor & & 1 & 4 & 8 & 12 & 16 & 20 & 32 \\
\hline BP & Subject 1 & 0.58 & 0.47 & 0.39 & 0.48 & 0.51 & 0.39 & 0.29 \\
& Subject 2 & 0.13 & 0.64 & 0.48 & 0.55 & 0.47 & 0.49 & 0.47 \\
& Subject 3 & 0.20 & 0.50 & 0.49 & 0.52 & 0.63 & 0.53 & 0.63 \\
WM & Subject 1 & 0.12 & 0.40 & 0.37 & 0.30 & 0.44 & 0.43 & 0.20 \\
& Subject 2 & 0.14 & 0.62 & 0.52 & 0.56 & 0.51 & 0.56 & 0.82 \\
& Subject 3 & 0.33 & 0.56 & 0.43 & 0.54 & 0.69 & 0.60 & 0.54 \\
GM & Subject 1 & 0.88 & 0.54 & 0.45 & 0.66 & 0.68 & 0.73 & 0.48 \\
& Subject 2 & 0.22 & 0.74 & 0.52 & 0.58 & 0.55 & 0.50 & 0.32 \\
& Subject 3 & 0.50 & 0.48 & 0.60 & 0.58 & 0.74 & 0.77 & 0.86 \\
CSF & Subject 1 & 0.88 & 0.53 & 0.41 & 0.77 & 0.69 & 0.84 & 0.37 \\
& Subject 2 & 0.29 & 0.54 & 0.43 & 0.42 & 0.49 & 0.43 & 0.37 \\
& Subject 3 & 0.61 & 0.19 & 0.33 & 0.28 & 0.43 & 0.50 & 0.63 \\
\hline
\end{tabular}

$C S$ compressed SENSE, $M D B$ md.brain segmentation and volumetry tool, $B P$ brain parenchyma, $W M$ white matter, $G M$ gray matter, $C S F$ cerebrospinal fluid vs. BP: $R^{2}=0.98$, NCR vs. WM: $R^{2}=0.98$, NCR vs. GM: $R^{2}=0.98$, NCR vs. CSF: $\left.R^{2}=0.98\right)$ than for FS-based volumes (NCR vs. BP: $R^{2}=0.81$, NCR vs. WM: $R^{2}=0.80, \mathrm{NCR}$ vs. GM: $R^{2}=0.94$, NCR vs. CSF: $R^{2}=0.94$ ), as visualized in Fig. 3b. This suggests that for MDB-based volumetry, the observed systematic CS-factor-dependent bias can be almost completely explained by the noise level increase induced by CS-acceleration. Analogously, for FS-based volumetry, the observed systematic CS-factor-dependent bias can, at least in large part, be explained by the noise level increase induced by CS-acceleration.

Additional analyses of smaller subcortical gray matter volumes of interest (pallidum, putamen, caudate and thalamus) resulted in higher $\mathrm{CoV}$ values compared to the analyzed whole-brain volumes BP, WM, GM and CSF (Supplementary Table 5).

\section{Reliability}

Except for the FS-based measurements at CS-factor $=32$, the narrow error bars in Fig. 2 give a good visual indication of the fairly low CoV. A CoV $<5 \%$ is considered acceptable [33]. Reliability assessed by ICC is considered to be poor $(<0.50)$, moderate $(0.50-0.75)$, good $(0.75-0.90)$ or excellent $(>0.90)$ [34].

\section{MDB-based volumetry}

For each CS-factor, the $\mathrm{CoV}$ of the repeatedly measured volumes was $<1 \%$ across all subjects and subvolumes (Table 6). ${ }^{1}$ Reliability assessed by ICC was good to excellent across all subvolumes up to CS-factor $=12$ (Table 7).

\section{FS-based volumetry}

For each CS-factor $<32$ and subvolumes BP, WM and GM, the $\mathrm{CoV}$ of the repeatedly measured volumes was $<1 \%$ across all subjects and subvolumes except for BP and GM

\footnotetext{
${ }^{1}$ One of the nine measurements at CS-factor $=16$ in subject 2 was detected as an outlier and excluded from the calculation of the CoV.
} 
at CS-factor $=16$ in subject $2(\text { Table } 8)^{1}$. For CS-factor $=32$, the repeatedly measured volumes showed significantly higher CoVs of up to 7\% for subvolumes BP, WM and GM. For CSF, the CoVs were even higher and reached up to $16 \%$ for CS-factor $<32$ and up to $25 \%$ for CS-factor $=32$ (Table 7). Reliability assessed by ICC was good to excellent across all subvolumes up to CS-factor $=12$ except for CSF which only showed a moderate reliability at CS-factor $=12$ (Table 9).

\section{Within-session and across-session reproducibility}

Mean and SD of the estimated brain volumes within a single session and across sessions for MDB and FS, respectively, can be found in the supplementary material. The results indicate that, except for CS-factors $>16$ and for the FS-based measurements of CSF, reproducibility is comparable when assessed across sessions as compared to within a single session.

\section{Discussion}

In our study, we showed that MRI acquisition acceleration via Compressed SENSE (CS) has a systematic bias effect on volumetric brain measurements which correlate strongly with image noise levels. This bias effect alters tissue quantification depending on the software used and analyzed subvolume. The present study is among the first to link a systematic volumetric bias of brain tissue with state of the art MR image acceleration techniques. The precision of the volumetric brain measurements remains high, even at a relatively high degree of acceleration.

For both software tools used, MDB and FS, we showed a systematic bias of the volumetric brain measurements proportional to the employed CS-factor. For both software tools and all analyzed subvolumes, this bias shows a strong linear behavior what was confirmed by linear correlation analysis $(R>0.95, p<0.01)$. This translates into increasing WM volumes for MDB and increasing CSF volumes for FS (Fig. 3). Considerable differences in intracranial tissue segmentation between SPM/CAT12 and FS are well known and have been demonstrated before. In particular, pronounced differences regarding inter-method and intra-method segmentations [35] as well as a dependence of inter-method variations in calculated volumes on the analyzed brain compartment [36] have been reported. In a recent study by Palumbo et al., the authors showed a systematic oversegmentation of GM volume by CAT 12 compared to FS [37]. This systematic difference in tissue classification between voxel-based morphometry and surface-based morphometry may be the reason for differences in absolute tissue volumes between MDB and FS.
Previous studies on parallel imaging techniques and brain volumes did not find a corresponding relationship investigating brain volumetry in healthy adults and dementia patients [38, 39]. However, these previous studies only investigated the effect of PI on volumetric brain measurements without additional compressed sensing. In our study, imaging data acquisition and processing was performed with NBV as the intended clinical application, i.e. for comparison of brain volumes with a normal cohort. For the rating of atrophy in dementia patients, providing additional quantitative information, for example, in the form of deviation maps or volume percentile curves, was shown to have a beneficial effect on diagnostic accuracy $[4,5]$. Although precise percentage cut-off values have not been defined, volumes below the fifth percentile or below two standard deviations are usually considered pathologic $[4,5]$. Knowing about systematic, CS-dependent tissue classification could lead to a more individual adjustment of these conventions for pathologic tissue atrophy, e.g. by means of a scaling factor. Since we demonstrated that the found systematic bias can vary across brain regions and the utilized analysis software, it may be reasonable to derive specific scaling factors depending on the respective setting. The alternative approach of matching CS-factors within the control cohort to the patient undergoing NBV evaluation seems not feasible since these cohorts are usually fixed.

NCR was calculated as quantitative measure of image quality and increased with the degree of acceleration as evidenced by the strong linear correlation with CS-factor. NCR and the calculated relative volumes also showed a strong linear correlation. This indicates that for MDBbased volumetry, and almost to the same extent for FSbased volumetry, the observed systematic bias is driven by the noise level increase induced by CS-acceleration. Furthermore, the enlarged views in Fig. 1 suggest that noise is more pronounced towards the image center (Fig. 1). Analyzing smaller subcortical regions of interest, we found less reliable results compared to whole-brain volumes BP, WM, GM and CSF. This supports the hypothesis of a nonuniform noise distribution with increased noise levels in the central regions. Previous studies about the relationship between image noise and measured brain volumes are scarce. In a reliability study of MRI measurements, Maclaren et al. [40] did not find any correlation between lateral ventricle volumes and image noise. However, the range of observed image noise was considerably narrower than in the previous study since the used sequences were not modified along the repeated measurements. The increased NCR is most likely the primary cause of the found CS-dependent systematic bias. Therefore, future investigations applying different denoising strategies to 
Table 7 Pairwise ICC to assess reliability between $\mathrm{CS}$-factor $=1$ and $\mathrm{CS}$-factor $=4,8,12,16,20$, 32 , respectively, for MDB

\begin{tabular}{lllllll}
\hline MDB & $\mathrm{ICC}_{1,4}$ & $\mathrm{ICC}_{1,8}$ & $\mathrm{ICC}_{1,12}$ & $\mathrm{ICC}_{1,16}$ & $\mathrm{ICC}_{1,20}$ & $\mathrm{ICC}_{1,32}$ \\
\hline $\mathrm{BP}$ & 0.9150 & 0.8760 & 0.7691 & 0.7027 & 0.6429 & 0.5412 \\
$\mathrm{WM}$ & 0.9758 & 0.9395 & 0.9187 & 0.8936 & 0.8762 & 0.7945 \\
$\mathrm{GM}$ & 0.9212 & 0.8948 & 0.7628 & 0.6880 & 0.6048 & 0.4747 \\
$\mathrm{CSF}$ & 0.9855 & 0.9841 & 0.9312 & 0.8613 & 0.7922 & 0.6356 \\
\hline
\end{tabular}

ICC intra-class correlation coefficient, $I C C_{l, i}$ ICC between CS-factors 1 and $I, C S$ compressed SENSE, $M D B$ md.brain software tool, $B P$ brain parenchyma, $W M$ white matter, $G M$ gray matter, $C S F$ cerebrospinal fluid

\begin{tabular}{llllllllr}
\hline CS-factor & & 1 & 4 & 8 & 12 & 16 & 20 & 32 \\
\hline BP & Subject 1 & 0.30 & 0.64 & 0.46 & 0.76 & 0.65 & 0.69 & 4.45 \\
& Subject 2 & 0.58 & 0.33 & 0.27 & 0.37 & 1.33 & 0.44 & 0.72 \\
& Subject 3 & 0.31 & 0.46 & 0.41 & 0.71 & 0.52 & 0.77 & 2.44 \\
WM & Subject 1 & 0.26 & 0.33 & 0.45 & 0.65 & 0.65 & 0.58 & 2.54 \\
& Subject 2 & 0.17 & 0.21 & 0.36 & 0.38 & 0.90 & 0.53 & 1.44 \\
& Subject 3 & 0.47 & 0.36 & 0.58 & 0.57 & 0.55 & 0.80 & 3.73 \\
GM & Subject 1 & 0.34 & 0.97 & 0.68 & 0.95 & 0.83 & 0.88 & 6.75 \\
& Subject 2 & 0.89 & 0.38 & 0.44 & 0.42 & 1.93 & 0.70 & 0.72 \\
& Subject 3 & 0.83 & 0.67 & 0.70 & 0.99 & 0.60 & 0.91 & 1.74 \\
CSF & Subject 1 & 7.80 & 8.15 & 4.76 & 8.68 & 10.53 & 11.77 & 24.47 \\
& Subject 2 & 4.47 & 5.19 & 6.24 & 3.15 & 14.89 & 8.88 & 7.68 \\
& Subject 3 & 3.58 & 5.30 & 9.67 & 9.36 & 7.09 & 4.15 & 14.45 \\
\hline
\end{tabular}

Table 8 Coefficients of variation $(\mathrm{CoV})$ in $\%$ of the FS-based repeated volumetric measurements

Table 9 Pairwise ICC to assess reliability between $\mathrm{CS}$-factor $=1$ and $\mathrm{CS}$-factor $=4,8,12,16,20$, 32, respectively, for FS

CS compressed SENSE, FS FreeSurfer segmentation and volumetry tool, BP brain parenchyma, WM white matter, $G M$ gray matter, $C S F$ cerebrospinal fluid

\begin{tabular}{lllllll}
\hline FS & $\mathrm{ICC}_{1,4}$ & $\mathrm{ICC}_{1,8}$ & $\mathrm{ICC}_{1,12}$ & $\mathrm{ICC}_{1,16}$ & $\mathrm{ICC}_{1,20}$ & $\mathrm{ICC}_{1,32}$ \\
\hline $\mathrm{BP}$ & 0.9996 & 0.9994 & 0.9987 & 0.9932 & 0.9940 & 0.9187 \\
$\mathrm{WM}$ & 0.9998 & 0.9992 & 0.9992 & 0.9968 & 0.9980 & 0.9946 \\
$\mathrm{GM}$ & 0.9993 & 0.9986 & 0.9928 & 0.9797 & 0.9661 & 0.6894 \\
$\mathrm{CSF}$ & 0.9550 & 0.8501 & 0.7233 & 0.6369 & 0.7014 & 0.3418 \\
\hline
\end{tabular}

ICC intra-class correlation coefficient, $I C C_{I, i}$ ICC between CS-factors 1 and $I, C S$ compressed SENSE, $F S$ FreeSurfer software tool, $B P$ brain parenchyma, $W M$ white matter, $G M$ gray matter, $C S F$ cerebrospinal fluid

the acquired MRI data prior to volumetric measurements could be a way to potentially reduce the biasing effect.

Except for FS-based volumetry of CSF, we have shown acceptable intra-subject reliability of the measured brain volumes using two different software tools. Interestingly, this holds true even for high acceleration factors up to CS-factor $=20$. In 2009, Lindholm et al. analyzed intrasubject reliability of brain volumetry accelerated by the PI technique GRAPPA. The reported CoVs for GM and WM have a comparable range $(0.4-1.6 \%)$ as the values found in our study [38]. One potential explanation for the preserved precision of measured brain volumes could be that the shortened acquisition time and associated reduction of motion artifacts compensates for the increased noise level at higher CS-factors. The low intrinsic measurement variability of CS-accelerated volumetric brain measurements found in our study can be considered a prerequisite for its application. Of note, no pre-processing for longitudinal brain volume evaluation was performed, since we focused on a single timepoint NBV scenario. This means that the observed $\mathrm{CoV}$ could potentially be reduced through 
optimized preprocessing steps for longitudinal brain volume assessment.

The present study is not without limitations. First, we only included three young healthy subjects. To validate our results in clinically relevant patient groups, further studies with a higher number of subjects, a broader age range and subjects suffering from neurodegenerative diseases are necessary. Additionally, these patients might be more prone to motion artifacts. Second, our study was primarily designed for the analysis of CS-acceleration in the context of NBV and not for the assessment of volume changes over time. To evaluate the impact on longitudinal brain volume analysis, a different study design and data processing would be needed. Third, for certain subvolumes, the found systematic bias shows marked differences between MDB and FS which cannot be explained in detail. To better comprehend the mechanism behind these differences, further investigations into the algorithms of the two software tools would be required but were beyond the scope of this study. Finally, the noise distribution of the imaging data was only assessed by visual inspection and the analysis of smaller subcortical volumes of interest in comparison to whole-brain volumes. Future studies should consider performing additional measurements on a uniform phantom image to improve the spatial characterization of image noise with regard to increasing acceleration factors.

\section{Conclusion}

We found that CS-accelerated MRI poses a systematic bias on measured brain volumes with differential effects on tissue classes depending on the volumetry pipeline used, at mostly preserved measurement precision. This bias effect leading to impaired accuracies of volume estimations is mostly explained by increasing image noise and should be taken into account when comparing brain volumes with external databases.

\begin{abstract}
Author contributions MD: study conception and design, acquisition of data, analysis and interpretation of data, drafting of manuscript, critical revision. AGR: analysis and interpretation of data. JS: acquisition of data. CW: analysis and interpretation of data, critical revision. LG: acquisition of data. JD: analysis and interpretation of data, critical revision. PFB: analysis and interpretation of data. AL: analysis and interpretation of data, critical revision. CZ: critical revision. JSK: critical revision. DMH: study conception and design, acquisition of data, analysis and interpretation of data, drafting of manuscript, critical revision.
\end{abstract}

Funding Open Access funding enabled and organized by Projekt DEAL.

\section{Compliance with ethical standards}

Conflict of interest The authors declare that they have no conflict of interest.

Ethical approval This article does not contain any studies with human participants or animals performed by any of the authors.

Open Access This article is licensed under a Creative Commons Attribution 4.0 International License, which permits use, sharing, adaptation, distribution and reproduction in any medium or format, as long as you give appropriate credit to the original author(s) and the source, provide a link to the Creative Commons licence, and indicate if changes were made. The images or other third party material in this article are included in the article's Creative Commons licence, unless indicated otherwise in a credit line to the material. If material is not included in the article's Creative Commons licence and your intended use is not permitted by statutory regulation or exceeds the permitted use, you will need to obtain permission directly from the copyright holder. To view a copy of this licence, visit http://creativecommons.org/licenses/by/4.0/.

\section{References}

1. Goodkin O, Pemberton H, Vos SB, Prados F, Sudre CH, Moggridge $J$ et al (2019) The quantitative neuroradiology initiative framework: application to dementia. Br J Radiol 92(1101):20190365

2. Giorgio A, De Stefano N (2013) Clinical use of brain volumetry. J Magn Reson Imaging 37(1):1-14

3. Kloppel S, Peter J, Ludl A, Pilatus A, Maier S, Mader I et al (2015) Applying automated MR-based diagnostic methods to the memory clinic: a prospective study. J Alzheimers Dis 47(4):939-954

4. Kloppel S, Yang S, Kellner E, Reisert M, Heimbach B, Urbach $\mathrm{H}$ et al (2018) Voxel-wise deviations from healthy aging for the detection of region-specific atrophy. Neuroimage Clin 20:851-860

5. Vernooij MW, Jasperse B, Steketee R, Koek M, Vrooman H, Ikram MA et al (2018) Automatic normative quantification of brain tissue volume to support the diagnosis of dementia: a clinical evaluation of diagnostic accuracy. Neuroimage Clin 20:374-379

6. Hedderich DM, Dieckmeyer M, Andrisan T, Ortner M, Grundl L, Schon $\mathrm{S}$ et al (2020) Normative brain volume reports may improve differential diagnosis of dementing neurodegenerative diseases in clinical practice. Eur Radiol 30(5):2821-2829

7. Benkarim OM, Sanroma G, Zimmer VA, Munoz-Moreno E, Hahner N, Eixarch E et al (2017) Toward the automatic quantification of in utero brain development in 3D structural MRI: a review. Hum Brain Mapp 38(5):2772-2787

8. Mugler JP 3rd, Brookeman JR (1991) Rapid three-dimensional T1-weighted MR imaging with the MP-RAGE sequence. J Magn Reson Imaging 1(5):561-567

9. OECD (2019) Magnetic resonance imaging (MRI) exams. https:// data.oecd.org/healthcare/magnetic-resonance-imaging-mri-exams .htm. Accessed 12 Dec 2020

10. Hollingsworth KG (2015) Reducing acquisition time in clinical MRI by data undersampling and compressed sensing reconstruction. Phys Med Biol 60(21):R297-322

11. Geerts-Ossevoort L, de Weerdt E, Duijndam A, van Ijperen G, Peeters H, Doneva M et al (2018) Compressed SENSE: speed done right. Every time. Philips FieldStrength Magazine, pp 1-16. https://philipsproductcontent.blob.core.windows.net/assets/20180 109/619119731f2a42c4acd4a863008a46c7.pdf. Accessed 12 Dec 2020 
12. Griswold MA, Jakob PM, Heidemann RM, Nittka M, Jellus V, Wang J et al (2002) Generalized autocalibrating partially parallel acquisitions (GRAPPA). Magn Reson Med 47(6):1202-1210

13. Bilgic B, Gagoski BA, Cauley SF, Fan AP, Polimeni JR, Grant PE et al (2015) Wave-CAIPI for highly accelerated 3D imaging. Magn Reson Med 73(6):2152-2162

14. Pruessmann KP, Weiger M, Scheidegger MB, Boesiger P (1999) SENSE: sensitivity encoding for fast MRI. Magn Reson Med 42(5):952-962

15. Donoho DL (2006) Compressed sensing. IEEE Trans Inf Theory 52(4):1289-1306

16. Lustig M, Donoho D, Pauly JM (2007) Sparse MRI: the application of compressed sensing for rapid MR imaging. Magn Reson Med 58(6):1182-1195

17. Monch S, Sollmann N, Hock A, Zimmer C, Kirschke JS, Hedderich DM (2019) Magnetic resonance imaging of the brain using compressed sensing - quality assessment in daily clinical routine. Clin Neuroradiol 30(2):279-286

18. Vranic JE, Cross NM, Wang Y, Hippe DS, de Weerdt E, MossaBasha M (2019) Compressed sensing-sensitivity encoding (CSSENSE) accelerated brain imaging: reduced scan time without reduced image quality. AJNR Am J Neuroradiol 40(1):92-98

19. Nielsen JA, Mair RW, Baker JT, Buckner RL (2019) Precision brain morphometry: feasibility and opportunities of extreme rapid scans. bioRxiv. https://doi.org/10.1101/530436

20. Longo MGF, Conklin J, Cauley SF, Setsompop K, Tian Q, Polak D et al (2020) Evaluation of ultrafast wave-CAIPI MPRAGE for visual grading and automated measurement of brain tissue volume. AJNR Am J Neuroradiol 41(8):1388-1396

21. LE Kurth F, Gaser C (2015) Voxel-based morphometry. Brain Mapp Encycl Ref 1:345-349

22. Ashburner J, Friston KJ (2000) Voxel-based morphometry-the methods. NeuroImage 11(6 Pt 1):805-821

23. Ashburner J, Friston KJ (2001) Why voxel-based morphometry should be used. NeuroImage 14(6):1238-1243

24. Ashburner JFK (2007) Voxel-based morphometry. In: Statistical parametric mapping: the analysis of functional brain images, $\mathrm{pp}$ 92-100. https://doi.org/10.1016/B978-0-12-372560-8.X5000-1

25. Dale AM, Fischl B, Sereno MI (1999) Cortical surface-based analysis. I. Segmentation and surface reconstruction. NeuroImage 9(2):179-194

26. FreeSurfer (2019). http://surfer.nmr.mgh.harvard.edu/. Accessed 12 Dec 2020

27. Fischl B, Salat DH, Busa E, Albert M, Dieterich M, Haselgrove $\mathrm{C}$ et al (2002) Whole brain segmentation: automated labeling of neuroanatomical structures in the human brain. Neuron 33(3):341-355
28. Fischl B, Salat DH, van der Kouwe AJ, Makris N, Segonne F, Quinn BT et al (2004) Sequence-independent segmentation of magnetic resonance images. NeuroImage 23(Suppl 1):S69-84

29. McGraw KO, Wong SP (1996) Forming inferences about some intraclass correlation coefficients. Psychol Methods 1(1):30-46

30. Liljequist D, Elfving B, Skavberg RK (2019) Intraclass correlation-a discussion and demonstration of basic features. PLoS ONE 14(7):e0219854

31. Gaser C (2019) CAT. http://www.neuro.uni-jena.de/cat. Accessed 12 Dec 2020

32. Grubbs FE (1950) Sample criteria for testing outlying observations. Ann Math Stat 21(1):27-58

33. Campbell MJ, Machin D, Walters SJ (2007) Medical statistics: a textbook for the health sciences, 4 th edn, ISBN: 978-0-470-02519-2

34. Koo TK, Li MY (2016) A guideline of selecting and reporting intraclass correlation coefficients for reliability research. J Chiropr Med 15(2):155-163

35. Klauschen F, Goldman A, Barra V, Meyer-Lindenberg A, Lundervold A (2009) Evaluation of automated brain MR image segmentation and volumetry methods. Hum Brain Mapp 30(4):1310-1327

36. Heinen R, Bouvy WH, Mendrik AM, Viergever MA, Biessels GJ, de Bresser J (2016) Robustness of automated methods for brain volume measurements across different MRI field strengths. PLoS ONE 11(10):e0165719

37. Palumbo L, Bosco P, Fantacci ME, Ferrari E, Oliva P, Spera G et al (2019) Evaluation of the intra- and inter-method agreement of brain MRI segmentation software packages: a comparison between SPM12 and FreeSurfer v6.0. Phys Med 64:261-272

38. Lindholm TL, Botes L, Engman EL, Frank A, Jonsson T, Svensson L et al (2009) Parallel imaging: is GRAPPA a useful acquisition tool for MR imaging intended for volumetric brain analysis? BMC Med Imaging 9:15

39. Gouttard S, Schaerer J, Yu H-J, Istace A, Roche F, Pachai C et al (2013) Impact of parallel imaging on volumetric brain MRI measurements in ADNI-GO and ADNI-2. Alzheimers Dement J Alzheimers Assoc 9(4):272

40. Maclaren J, Han Z, Vos SB, Fischbein N, Bammer R (2014) Reliability of brain volume measurements: a test-retest dataset. Sci Data 1:140037

Publisher's Note Springer Nature remains neutral with regard to jurisdictional claims in published maps and institutional affiliations. 